\title{
A Convergence Theorem Associated With a Pair of Second Order Differential Equations
}

\author{
Amar Kumar \\ J R S College, Jamalpur (T M Bhagalpur University, Bhagalpur)
}

\begin{abstract}
We consider the second order matrix differential equation

$$
(M+\lambda) \Phi=0,0 \leq x<\infty .
$$

Where $M$ is a second-order matrix differential operator and $\Phi$ is a vector having two components. In this paper we prove a convergence theorem for the vector function $f(x)=\left[\begin{array}{l}f_{1}(x) \\ f_{2}(x)\end{array}\right]$ which is continuous in $0 \leq x<\infty$ and of bounded variation in $0 \leq x<\infty$, when $p(x)$ and $q(x)$ tend to $-\infty$ as $x$ tend to $+\infty$.

Key Words: Matrix differential operator, convergence theorem, bounded variation.
\end{abstract}

\$1. Let $M$ denote the matrix operator

$$
M=\left[\begin{array}{cc}
\frac{d^{2}}{d x^{2}}-p(x) & r(x) \\
r(x) & \frac{d^{2}}{d x^{2}}-q(x)
\end{array}\right]
$$

and $\Phi=\Phi(x)$ a vector having two components $u=u(x)$ and $v=v(x)$ represented as a column matrix

Consider the homogenous system

$$
\Phi=\left[\begin{array}{l}
u \\
v
\end{array}\right] .
$$

$$
(M+\lambda) \Phi=0, \quad 0 \leq x<\infty .
$$

where $\lambda$ is a parameter, real or complex.

We assume the following conditions to be satisfied:

(i) $\quad p(x), q(x)$ tend to $-\infty$ as $x$ tend to $+\infty$.

(ii) $\quad p^{\prime}(x) \leq 0, q^{\prime}(x) \leq 0, p^{\prime \prime}(x) \leq 0, q^{\prime \prime}(x) \leq 0$.

(iii) $\quad p^{\prime}(x)=\circ\left[(p(x))^{c}\right], 0<c<\frac{3}{2}$.

(iv) $\quad q^{\prime}(x)=\circ\left[(q(x))^{c_{1}}\right], 0<c_{1}<\frac{3}{2}$.

(v) $\quad r(x)$ is bounded or $r(x)=\circ\left[(p(x) q(x))^{d}\right], 0<d<\frac{1}{4}$.

$$
\int_{0}^{\infty}(p(x))^{-\frac{1}{2}} d x \text { and } \int_{0}^{\infty}(q(x))^{-\frac{1}{2}} d x \text { are divergent. }
$$

$$
\int_{0}^{\infty}(p(x))^{-\frac{1}{2}} d x \square \int_{0}^{\infty}(q(x))^{-\frac{1}{2}} d x \text { is convergent as } x \rightarrow \infty .
$$

Following Bhagat [2], the bilinear concomitant $[\Phi \theta]$ of two vectors

is defined by

$$
\Phi=\left[\begin{array}{l}
\varphi_{1} \\
\varphi_{2}
\end{array}\right] \text { and } \theta=\left[\begin{array}{l}
\vartheta_{1} \\
\vartheta_{2}
\end{array}\right]
$$

$$
[\Phi \theta]=\varphi_{1}^{\prime} \vartheta_{1}-\varphi_{1} \vartheta_{1}^{\prime}+\varphi_{2}^{\prime} \vartheta_{2}-\varphi_{2} \vartheta_{2}^{\prime}
$$




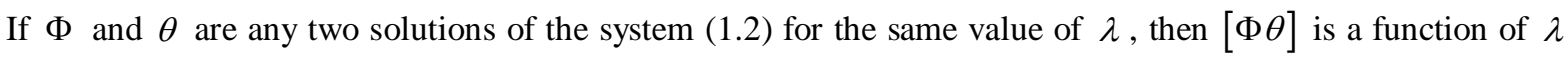
alone. It is an integral function of $\lambda$, real for real $\lambda$ (see Bhagat [1]).

Let $\varphi_{j}(x, \lambda) \equiv \varphi_{j}(0 / x ; \lambda)=\left[\begin{array}{l}u_{j}(0 / x ; \lambda) \\ v_{j}(0 / x ; \lambda)\end{array}\right],(j=1,2)$ be the boundary condition vectors at $x=0$ by

$$
\left.\begin{array}{ll}
u_{j}(0 / x ; \lambda)=a_{j 2} & u_{j}^{\prime}(0 / x ; \lambda)=-a_{j 1} \\
v_{j}(0 / x ; \lambda)=a_{j 4} & v_{j}^{\prime}(0 / x ; \lambda)=-a_{j 3}
\end{array}\right\}, \quad(j=1,2),
$$

so that the boundary conditions to be satisfied by any solution $\varphi(x, \lambda)=\left[\begin{array}{l}u(x, \lambda) \\ v(x, \lambda)\end{array}\right]$ of (1.2) at $x=0$ are given by

$$
\left[\varphi(x, \lambda) \varphi_{j}(x, \lambda)\right]=0,(j=1,2)
$$

and

$$
\left[\begin{array}{ll}
\varphi_{1} & \varphi_{2}
\end{array}\right]=0
$$

The vectors $\vartheta_{k}(x, \lambda) \equiv \vartheta_{k}(0 / x ; \lambda)=\left[\begin{array}{l}x_{k}(0 / x ; \lambda) \\ y_{k}(0 / x ; \lambda)\end{array}\right], \quad(k=1,2)$ which take real constant values (independent of $\lambda)$ at $x=0$ are defined by the relations

$$
\left[\begin{array}{ll}
\varphi_{j} & \vartheta_{k}
\end{array}\right]=\delta_{j k},\left[\begin{array}{ll}
\vartheta_{1} & \vartheta_{2}
\end{array}\right]=0 \quad(1 \leq j, k \leq 2)
$$

\section{\$2. Green Matrix:}

The Green matrix $G(x, y ; \lambda)=\left[\begin{array}{ll}G_{11} & G_{21} \\ G_{12} & G_{22}\end{array}\right]$ for the system (1.2) is given by

$$
\begin{aligned}
G_{1}(x, y ; \lambda) & =\left[\begin{array}{ll}
\psi_{11}(x, \lambda) & \psi_{21}(x, \lambda) \\
\psi_{12}(x, \lambda) & \psi_{22}(x, \lambda)
\end{array}\right]\left[\begin{array}{ll}
u_{1}(y, \lambda) & v_{1}(y, \lambda) \\
u_{2}(y, \lambda) & v_{2}(y, \lambda)
\end{array}\right] ; y \in[0, x) \\
& =\left[\begin{array}{ll}
u_{1}(x, \lambda) & v_{1}(x, \lambda) \\
u_{2}(x, \lambda) & v_{2}(x, \lambda)
\end{array}\right]\left[\begin{array}{ll}
\psi_{11}(y, \lambda) & \psi_{21}(y, \lambda) \\
\psi_{12}(y, \lambda) & \psi_{22}(y, \lambda)
\end{array}\right] ; y \in(x, \infty)
\end{aligned}
$$

We shall use the notations and results of Bhagat [3] and Pandey and Kumar [6]. The method of Titchmarsh [8] will be used to obtain the results analogous to [7].

\$3. Let $A_{j}(x, \lambda)=\left[\begin{array}{l}S_{j}(x, \lambda) \\ T_{j}(x, \lambda)\end{array}\right],(j=1,2)$. It can be verified following Titehmarsh $[8, \S 5.4]$ that $A_{j}(x, \lambda)$ satisfies the system of integral equations.

$$
\left.\begin{array}{c}
S_{j}(x, \lambda)=S_{j}(0) \cos w(x)+\frac{1}{\mu} S_{j}^{\prime}(0) \sin w(x)-\int_{0}^{x}\left[P(t) S_{j}(t, \lambda)+R(t) T_{j}(t, \lambda)\right] \sin (w(x)-w(t)) d t \\
T_{j}(x, \lambda)=T_{j}(0) \cos z(x)+\frac{1}{\mu} T_{j}^{\prime}(0) \sin z(x)-\int_{0}^{x}\left[Q(t) T_{j}(t, \lambda)+R(t) S_{j}(t, \lambda)\right] \sin (z(x)-z(t)) d t
\end{array}\right\},(j=1,2)(3.1)
$$

When $\lambda=\mu^{2}$, then

$$
\begin{aligned}
& S_{j}(x, \lambda)=(\lambda-p(x))^{\frac{1}{4}} u_{j}(x, \lambda), \quad(j=1,2) \\
& T_{j}(x, \lambda)=(\lambda-q(x))^{\frac{1}{4}} v_{j}(x, \lambda), \quad(j=1,2) \\
& w(x)=\int_{0}^{x}(\lambda-p(t))^{\frac{1}{2}} d t \\
& z(x)=\int_{0}^{x}(\lambda-q(t))^{\frac{1}{2}} d t \\
& P(x)=\frac{1}{4} \frac{p^{\prime \prime}(x)}{(\lambda-p(x))^{\frac{3}{2}}}+\frac{5}{16} \frac{\left(p^{\prime}(x)\right)^{2}}{(\lambda-p(x))^{\frac{5}{2}}} \\
& Q(x)=\frac{1}{4} \frac{q^{\prime \prime}(x)}{(\lambda-q(x))^{\frac{3}{2}}}+\frac{5}{16} \frac{\left(q^{\prime}(x)\right)^{2}}{(\lambda-q(x))^{\frac{5}{2}}}
\end{aligned}
$$




$$
R(x)=\frac{r(x)}{(\lambda-p(x))^{\frac{1}{4}}(\lambda-q(x))^{\frac{1}{4}}}
$$

We assume that $p(x)$ and $q(x)$ are bounded for all finite $x$ and $p(0)=q(0)=0$. So for a fixed $x$ and large $|\lambda|$, we have from (3.2) - (3.8)

$$
\begin{aligned}
& S_{j}(0)=(\lambda)^{\frac{1}{4}} u_{j}(0), \quad(j=1,2) \\
& T_{j}(0)=(\lambda)^{\frac{1}{4}} v_{j}(0), \quad(j=1,2) \\
& S_{j}^{\prime}(0)=(\lambda)^{\frac{1}{4}} u_{j}^{\prime}(0)+\circ\left(|\lambda|^{-\frac{3}{4}}\right), \quad(j=1,2) \\
& T_{j}^{\prime}(0)=(\lambda)^{\frac{1}{4}} v_{j}^{\prime}(0)+\circ\left(|\lambda|^{-\frac{3}{4}}\right), \quad(j=1,2) \\
& w(x)=\lambda^{\frac{1}{2}}+\circ\left(|\lambda|^{-\frac{1}{2}}\right) \\
& z(x)=\lambda^{\frac{1}{2}}+\circ\left(|\lambda|^{-\frac{1}{2}}\right) \\
& (\lambda-p(x))^{\frac{1}{4}}=(\lambda)^{\frac{1}{4}}+\circ\left(|\lambda|^{-\frac{3}{4}}\right) \\
& (\lambda-q(x))^{\frac{1}{4}}=(\lambda)^{\frac{1}{4}}+\circ\left(|\lambda|^{-\frac{3}{4}}\right) \\
& P(x)=\circ\left(|\lambda|^{-\frac{3}{4}}\right) \\
& Q(x)=\circ\left(|\lambda|^{-\frac{3}{4}}\right) \\
& R(x)=\circ\left(|\lambda|^{-\frac{1}{2}}\right) \\
& E \mu
\end{aligned}
$$

Let $\mu=s+i t, t>0$. Therefore,

Therefore, from (3.1), we have

$$
\left.\begin{array}{l}
S_{j}(x, \lambda)=H_{j 1} \cdot e^{t . x} \\
T_{j}(x, \lambda)=H_{j 2} \cdot e^{t . x}
\end{array}\right\}, \quad(j=1,2)
$$

$$
\left.\begin{array}{rl}
H_{j 1}(x, \lambda)= & {\left[S_{j}(0) \cos w(x)+\frac{1}{\mu} S_{j}^{\prime}(0) \sin w(x)\right] \cdot e^{-t x}-} \\
& -\int_{0}^{x} e^{-t(x-y)}\left[P(y) H_{j 1}(y, \lambda)+R(y) H_{j 2}(y, \lambda)\right] \sin (w(x)-w(y)) d y \\
H_{j 2}(x, \lambda)= & {\left[T_{j}(0) \cos z(x)+\frac{1}{\mu} T_{j}^{\prime}(0) \sin z(x)\right] \cdot e^{-t x}-} \\
& -\int_{0}^{x} e^{-t(x-y)}\left[Q(y) H_{j 2}(y, \lambda)+R(y) H_{j 1}(y, \lambda)\right] \sin (z(x)-z(y)) d y
\end{array}\right\}
$$

Let

$$
\left.\begin{array}{c}
M=\max \left[S_{j}(0), T_{j}(0), S_{j}^{\prime}(0), T_{j}^{\prime}(0)\right] \\
N(y)=\max [|P(y)|,|Q(y)|,|R(y)|]
\end{array}\right\}
$$

Now we have

$$
\text { and } \left.\begin{array}{r}
|\cos w(x)|,|\sin w(x)| \leq e^{t x} \\
|\sin z(x)|,|\cos z(x)| \leq e^{t x}
\end{array}\right\} \text { for large }|\lambda|
$$

Therefore, using (3.22) and (3.23), (3.21) gives 


$$
H_{j 1}(x, \lambda), H_{j 2}(x, \lambda) \leq M\left(1+\frac{1}{|\mu|}\right) \times \int_{0}^{x}\left\{\left|H_{j 1}(y, \lambda)\right|,\left|H_{j 2}(y, \lambda)\right|\right\} \cdot N(y) d y \text { for large }|\lambda| .
$$

Therefore from Conte and Sangren Lemma of [14], we have

$$
\left|H_{j 1}(y, \lambda)\right|,\left|H_{j 2}(y, \lambda)\right| \leq M\left(1+\frac{1}{|\mu|}\right) \cdot \exp \left\{2 \int_{0}^{x} N(y) d y\right\}, \quad(j=1,2)
$$

Thus, we see that $H_{j 1}$ and $H_{j 2}$ are bounded for all $x$ and large $|\lambda|$. It follows from (3.20) that

$$
S_{j}(x, \lambda), T_{j}(x, \lambda)=o\left(e^{t x}\right), \quad(j=1,2)
$$

From (3.1), using (3.25)

$$
\text { for all } x \text { and large }|\lambda| \text {. }
$$

$$
\left.\begin{array}{l}
S_{j}(x, \lambda)=S_{j}(0) \cos w(x)+\circ\left(e^{t x} \cdot|\lambda|^{-\frac{1}{2}}\right) \\
T_{j}(x, \lambda)=T_{j}(0) \cos z(x)+\circ\left(e^{t x} \cdot|\lambda|^{-\frac{1}{2}}\right)
\end{array}\right\}, \quad(j=1,2)
$$

Using (3.2) and (3.3), we get from (3.26)

$$
\left.\begin{array}{l}
u_{j}(x, \lambda)=u_{j}(0) \cos w(x)+\circ\left(e^{t x} \cdot|\lambda|^{-\frac{3}{4}}\right) \\
v_{j}(x, \lambda)=v_{j}(0) \cos z(x)+\circ\left(e^{t x} \cdot|\lambda|^{-\frac{3}{4}}\right)
\end{array}\right\}, \quad(j=1,2)
$$

Also, we have from [5, Chap.3, §4], for large $x$.

where

$$
\begin{aligned}
& u_{j}(x, \lambda)=\frac{e^{-i w(x)}\left[M_{j 1}(\lambda)+o(1)\right]}{(\lambda-p(x))^{\frac{1}{4}}}, \quad(j=1,2) \\
& v_{j}(x, \lambda)=\frac{e^{-i z(x)}\left[M_{j 2}(\lambda)+o(1)\right]}{(\lambda-q(x))^{\frac{1}{4}}}, \quad(j=1,2)
\end{aligned}
$$

$$
\begin{aligned}
M_{j 1}(\lambda)= & \frac{1}{2} \lambda^{\frac{1}{4}} u_{j}(0)-\frac{1}{2 i}\left(\frac{u_{j}^{\prime}(0)}{\lambda^{\frac{1}{4}}}-\frac{u_{j}(0) p^{\prime}(0)}{4 \lambda^{\frac{5}{4}}}\right)+ \\
& +\frac{1}{2 i} \int_{0}^{\infty} e^{i w(t)}\left\{P(t)(\lambda-p(t))^{\frac{1}{4}} u_{j}(t, \lambda)+R(t)(\lambda-q(t))^{\frac{1}{4}} v_{j}(t, \lambda)\right\} d t \\
M_{j 2}(\lambda)= & \frac{1}{2} \lambda^{\frac{1}{4}} v_{j}(0)-\frac{1}{2 i}\left(\frac{v_{j}^{\prime}(0)}{\lambda^{\frac{1}{4}}}-\frac{v_{j}(0) q^{\prime}(0)}{4 \lambda^{\frac{5}{4}}}\right)+ \\
& +\frac{1}{2 i} \int_{0}^{\infty} e^{i z(t)}\left\{Q(t)(\lambda-q(t))^{\frac{1}{4}} v_{j}(t, \lambda)+R(t)(\lambda-p(t))^{\frac{1}{4}} u_{j}(t, \lambda)\right\} d t
\end{aligned}
$$

under the condition $\operatorname{im}(w(x) \square z(x))=\mathrm{o}(1)$.

\$4. In this section we obtain a solution of the system (1.2) which is small when imaginary part of $\lambda$ is large and positive and $x$ is large. To find such a solution we consider the system of integral equations 


$$
\begin{aligned}
X_{j}(x, \lambda)= & \left.e^{i w(x)}-\frac{1}{2 i} \int_{0}^{x} e^{i(w(x)-w(t))}\left\{P(t) X_{j}(t, \lambda)+R(t) Y_{j}(t, \lambda)\right\} d t-\right\} \\
& -\frac{1}{2 i} \int_{x}^{\infty} e^{i(w(x)-w(t))}\left\{P(t) X_{j}(t, \lambda)+R(t) Y_{j}(t, \lambda)\right\} d t \\
Y_{j}(x, \lambda)= & \left.e^{i z(x)}-\frac{1}{2 i} \int_{0}^{x} e^{i(z(x)-z(t))}\left\{Q(t) Y_{j}(t, \lambda)+R(t) X_{j}(t, \lambda)\right\} d t-\right\}, \quad(j=1,2) \\
& -\frac{1}{2 i} \int_{x}^{\infty} e^{i(z(x)-z(t))}\left\{Q(t) Y_{j}(t, \lambda)+R(t) X_{j}(t, \lambda)\right\} d t
\end{aligned}
$$

Exactly following Titchmarsh $[8, \S 6.2]$ and using (vii) of $\S 3$ it can be verified that the solutions of the system of integral equations (4.1) satisfying (1.2). Also we have

$$
\left.\begin{array}{l}
\left|X_{j}(x, \lambda)\right| \leq \frac{e^{-i w(x)}}{(1-J)} \\
\left|Y_{j}(x, \lambda)\right| \leq \frac{e^{-i z(x)}}{(1-J)}
\end{array}\right\}, \quad(j=1,2)
$$

where

$$
J=\max \left[\int_{0}^{\infty}|P(y)| d y, \int_{0}^{\infty}|Q(y)| d y, \int_{0}^{\infty}|R(y)| e^{i m(w(y)-z(y))} d y, \int_{0}^{\infty}|R(y)| e^{i m(z(y)-w(y))} d y\right]
$$

Considering (4.1) for a fixed $\lambda$ or $\lambda$ in the bounded part of the region $J=\circ\left(|\lambda|^{-\frac{1}{2}}\right)<1$, if $|\lambda|$ is sufficiently large and noting that $i m(w(x)-z(x))=\circ(1)$, it can be shown following [8, §6.2] that

$$
\left.\begin{array}{l}
X_{j}(x, \lambda)=e^{i w(x)}\left[L_{j 1}(\lambda)+o(1)\right] \\
Y_{j}(x, \lambda)=e^{i z(x)}\left[L_{j 2}(\lambda)+o(1)\right]
\end{array}\right\}, \quad(j=1,2)
$$

where

$$
\begin{aligned}
& L_{j 1}(\lambda)=1-\frac{1}{2 i} \int_{0}^{\infty} e^{-i w(y)}\left\{P(y) X_{j}(y, \lambda)+R(y) Y_{j}(y, \lambda)\right\} d y \\
& \left.Y_{j}(x, \lambda)=1-\frac{1}{2 i} \int_{0}^{\infty} e^{-i z(y)}\left\{Q(y) Y_{j}(y, \lambda)+R(y) X_{j}(y, \lambda)\right\} d y\right\}, \quad(j=1,2)
\end{aligned}
$$

From (3.2), (3.3) and (3.4), we have

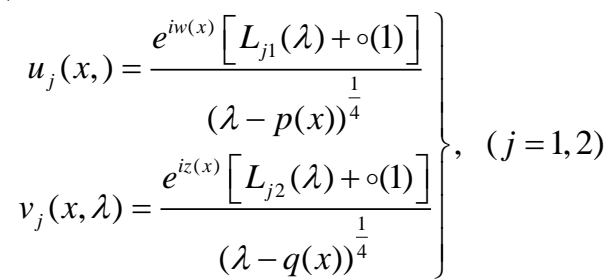

\$5. From (3.28) and $(3,29)$ we see that $\varphi_{j}(x, \lambda),(j=1,2)$ are large when the imaginary part of $w(x)$ and $z(x)$ are large and positive. Therefore $\varphi_{j}(x, \lambda),(j=1,2)$ are not $L^{2}[0, \infty)$. But from (4.5) we see that $\alpha_{j}(x, \lambda)=\left[\begin{array}{l}u_{j}(x, \lambda) \\ v_{j}(x, \lambda)\end{array}\right], \quad(j=1,2)$

are small when the imaginary part of $w(x)$ and $z(x)$ are large and positive. Thus $\varphi_{j}(x, \lambda)$ and $\alpha_{j}(x, \lambda)$, $(j=1,2)$ are linearly independent. Then

$$
\psi_{r}(x, \lambda)=\sum_{s=1}^{2} K_{r s}(\lambda) \alpha_{s}(x, \lambda)+\sum_{s=1}^{2} L_{r s}(\lambda) \varphi_{s}(x, \lambda), \quad(r=1,2)
$$

Since $\psi_{r}(x, \lambda), \quad(r=1,2)$ are $L^{2}[0, \infty)$ but $\varphi_{j}(x, \lambda)$ are not $L^{2}[0, \infty)$, therefore $L_{r s}(\lambda)=0, \quad(1 \leq r, s \leq 2)$. Hence 


$$
\psi_{r}(x, \lambda)=\sum_{s=1}^{2} K_{r s}(\lambda) \alpha_{s}(x, \lambda), \quad(r=1,2)
$$

From asymptotic formulae (3.28), (3.29) and (4.3) we have, as $x$ tend to infinity

$$
\left.\begin{array}{c}
u_{j}^{\prime}(x, \lambda) \square-i(\lambda-p(x))^{\frac{1}{4}} e^{-i w(x)} M_{j 1}(\lambda) \\
v_{j}^{\prime}(x, \lambda) \square-i(\lambda-q(x))^{\frac{1}{4}} e^{-i z(x)} M_{j 2}(\lambda) \\
u_{j}^{\prime}(x, \lambda) \square-i(\lambda-p(x))^{\frac{1}{4}} e^{i w(x)} L_{j 1}(\lambda) \\
v_{j}^{\prime}(x, \lambda) \square-i(\lambda-q(x))^{\frac{1}{4}} e^{-i z(x)} L_{j 1}(\lambda)
\end{array}\right\}, \quad(j=1,2)
$$

where dashes denote differentiation with respect to $x$. Using (3.28), (3.29), (4.5), (5.2) and (5.3) we obtain from (1.5)

$$
\left.\begin{array}{l}
K_{11}(\lambda)=\frac{M_{21} L_{21}+M_{22} L_{22}}{2 i\left(M_{11} M_{22}-M_{12} M_{21}\right)\left(L_{12} L_{21}-L_{11} L_{22}\right)} \\
K_{12}(\lambda)=\frac{M_{21} L_{11}+M_{22} L_{12}}{2 i\left(M_{11} M_{22}-M_{12} M_{21}\right)\left(L_{12} L_{21}-L_{11} L_{22}\right)} \\
K_{21}(\lambda)=\frac{M_{11} L_{21}+M_{12} L_{22}}{2 i\left(M_{11} M_{22}-M_{12} M_{21}\right)\left(L_{12} L_{21}-L_{11} L_{22}\right)} \\
K_{22}(\lambda)=\frac{M_{11} L_{11}+M_{12} L_{12}}{2 i\left(M_{11} M_{22}-M_{12} M_{21}\right)\left(L_{12} L_{21}-L_{11} L_{22}\right)}
\end{array}\right\}
$$

\section{\$6. Convergence Theorem:}

If $f(x)=\left[\begin{array}{l}f_{1}(x) \\ f_{2}(x)\end{array}\right]$ be a real valued continuous vector of bounded variation in $0 \leq x<\infty$, and $L^{2}[0, \infty)$ and is such that the integrals

$$
\int_{0}^{\infty} p(x) f_{1}(x) d x \quad ; \int_{0}^{\infty} p(x) f_{1}(x) d x
$$

are uniformly convergent for large $|\lambda|$, then

uniformly for $\quad 0<\varepsilon \leq 1$, where

$$
f(x)=-\frac{1}{\pi i} \lim _{R \rightarrow \infty} \int_{-R+i \varepsilon}^{R+i \varepsilon} \varphi(x, \lambda) d \lambda
$$

$$
\left[\begin{array}{l}
\varphi_{1}(x, \lambda) \\
\varphi_{2}(x, \lambda)
\end{array}\right]=\varphi(x, \lambda)=\int_{0}^{\infty} G(x, y ; \lambda) f(y) d y
$$

We prove convergence theorem for $\varphi_{1}(x, \lambda)$ because similar result holds for $\varphi_{2}(x, \lambda)$.

Now we write $\varphi_{1}(x, \lambda)$ as

$$
\begin{aligned}
\varphi_{1}(x, \lambda)= & \psi_{11}(x, \lambda) \int_{0}^{x} \varphi_{1}^{T}(y, \lambda) f(y) d y+\psi_{21}(x, \lambda) \int_{0}^{x} \varphi_{2}^{T}(y, \lambda) f(y) d y+ \\
& +u_{1}(x, \lambda) \int_{x}^{\infty} \psi_{1}^{T}(y, \lambda) f(y) d y+u_{2}(x, \lambda) \int_{x}^{\infty} \psi_{2}^{T}(y, \lambda) f(y) d y . \\
\varphi_{1}(x, \lambda)= & \psi_{11}(x, \lambda) \int_{0}^{x} u_{1}(y, \lambda) f_{1}(y) d y+u_{1}(x, \lambda) \int_{x}^{\infty} \psi_{11}(y, \lambda) f_{1}(y) d y+\psi_{21}(x, \lambda) \int_{0}^{x} u_{2}(y, \lambda) f_{1}(y) d y+ \\
& +u_{2}(x, \lambda) \int_{x}^{\infty} \psi_{21}(y, \lambda) f_{1}(y) d y+\psi_{11}(x, \lambda) \int_{0}^{x} v_{1}(y, \lambda) f_{2}(y) d y+\psi_{21}(x, \lambda) \int_{0}^{x} v_{2}(y, \lambda) f_{2}(y) d y+ \\
& +u_{1}(x, \lambda) \int_{x}^{\infty} \psi_{12}(y, \lambda) f_{2}(y) d y+u_{2}(x, \lambda) \int_{x}^{\infty} \psi_{22}(y, \lambda) f_{2}(y) d y . \\
& =A+B+C+D+E+F
\end{aligned}
$$

where 


$$
\begin{aligned}
& A=\psi_{11}(x, \lambda) \int_{0}^{x} u_{1}(y, \lambda) f_{1}(y) d y+u_{1}(x, \lambda) \int_{x}^{\infty} \psi_{11}(y, \lambda) f_{1}(y) d y \\
& B=\psi_{21}(x, \lambda) \int_{0}^{x} u_{2}(y, \lambda) f_{1}(y) d y+u_{2}(x, \lambda) \int_{x}^{\infty} \psi_{21}(y, \lambda) f_{1}(y) d y \\
& C=\psi_{11}(x, \lambda) \int_{0}^{x} v_{1}(y, \lambda) f_{2}(y) d y \\
& D=\psi_{21}(x, \lambda) \int_{0}^{x} v_{2}(y, \lambda) f_{2}(y) d y \\
& E=u_{1}(x, \lambda) \int_{x}^{\infty} \psi_{12}(y, \lambda) f_{2}(y) d y \\
& F=u_{2}(x, \lambda) \int_{x}^{\infty} \psi_{22}(y, \lambda) f_{2}(y) d y .
\end{aligned}
$$

We evaluate $A$, the other term can be evaluated in the same way. Now

$$
\begin{aligned}
& A=\psi_{11}(x, \lambda) \int_{0}^{x} u_{1}(y, \lambda) f_{1}(y) d y+u_{1}(x, \lambda) \int_{x}^{\infty} \psi_{11}(y, \lambda) f_{1}(y) d y \\
& =\psi_{11}(x, \lambda)\left[\int_{0}^{x-\delta}+\int_{x-\delta}^{x} u_{1}(y, \lambda) f_{1}(y) d y\right]+u_{1}(x, \lambda)\left[\int_{x}^{x+\delta} \int_{x+\delta}^{\infty} \psi_{11}(y, \lambda) f_{1}(y) d y\right] \\
& =A_{1}+A_{2}+A_{3}+A_{4} \text {, say }
\end{aligned}
$$

For $J<1$, if $|\lambda|$ is sufficiently large, we have from (5.2) and (5.4)

$$
\begin{aligned}
\left|\psi_{11}(x, \lambda)\right| & \leq \frac{\left|M_{22}(\lambda)\right|\left|e^{i w(x)}\right|}{2\left\{\left|M_{11}(\lambda) M_{22}(\lambda)-M_{12}(\lambda) M_{21}(\lambda)\right|\right\}|\lambda-p(x)|^{\frac{1}{4}}} \times \\
& \times \frac{\left|M_{22}(\lambda)\right| e^{-i m(w(x))}}{2\left\{\left|M_{11}(\lambda) M_{22}(\lambda)-M_{12}(\lambda) M_{21}(\lambda)\right|\right\} \lambda^{\frac{1}{4}}\left[1-\frac{p(x)}{\lambda}\right]^{\frac{1}{4}}}
\end{aligned}
$$

Therefore, using (3.2), (3.25) and (6.5), we have

$$
A_{4}=o\left\{\frac{e^{t x}}{|\lambda|^{\frac{1}{2}}} \int_{x+\delta}^{\infty} e^{-t y}\left|f_{1}(y)\right| d y\right\}
$$

For a fixed $y, p(y)$ is less than $|\lambda|$. Therefore, using (6.1), we have

$$
A_{4}=o\left\{\frac{e^{-t \delta}}{|\lambda|^{\frac{1}{2}}}\right\}
$$

The integral of (6.6) round the semicircle tends to zero as $R$ tends to infinity for any fixed $\delta>0$. A similar argument holds for $A_{1}$ also. Now, we consider $A_{3}$. For fixed $x$ or in a finite interval, from (4.1), we have

$$
\begin{aligned}
& \left|X_{j}(x, \lambda)-e^{i w(x)}\right|=\left|\frac{1}{2 i} \int_{0}^{x} e^{i(w(x)-w(y))}\left\{P(y) X_{j}(y, \lambda)+R(y) Y_{j}(y, \lambda)\right\} d y+\frac{1}{2 i} \int_{x}^{\infty} e^{i(w(y)-w(x))}\left\{P(y) X_{j}(y, \lambda)+R(y) Y_{j}(y, \lambda)\right\} d y\right| \\
& \leq \frac{e^{-i m(w(x))}}{2}(J+---) \\
& <\alpha \cdot e^{-i m(w(x))}, \quad(j=1,2) \quad \text { (say) }
\end{aligned}
$$

Similarly,

$$
\left|Y_{j}(x, \lambda)-e^{i z(x)}\right|<\alpha \cdot e^{-i m(z(x))}, \quad(j=1,2) \quad \text { (say) }
$$

Also from (4.4), we have 


$$
\begin{aligned}
& \left.L_{j 1}(\lambda)=1+\circ\left(|\lambda|^{-\frac{1}{2}}\right)\right) \\
& \left.L_{j 2}(\lambda)=1+\circ\left(|\lambda|^{-\frac{1}{2}}\right)\right\}, \quad(j=1,2)
\end{aligned}
$$

Similarly from (3.30) and (3.31), we have

$$
\begin{aligned}
& M_{j 1}(\lambda)=\frac{1}{2} \lambda^{\frac{1}{4}} u_{j}(0)+\circ\left(|\lambda|^{-\frac{1}{4}}\right) \\
& \left.M_{j 2}(\lambda)=\frac{1}{2} \lambda^{\frac{1}{4}} v_{j}(0)+\circ\left(|\lambda|^{-\frac{1}{4}}\right)\right\}, \quad(j=1,2)
\end{aligned}
$$

Therefore, by using (6.9), (4.3) can be written as

$$
\begin{aligned}
& \left.X_{j}(x, \lambda)=e^{i w(x)}\left[1+\circ\left(|\lambda|^{-\frac{1}{2}}\right)\right]\right], \quad(j=1,2) \\
& Y_{j}(x, \lambda)=e^{i z(x)}\left[1+\circ\left(|\lambda|^{-\frac{1}{2}}\right)\right]
\end{aligned}
$$

Now using (4.5), (6.10) and (6.11), (5.2) and (5.4) give

$$
\begin{gathered}
\psi_{11}(x, \lambda)=\frac{v_{2}(0) \cdot e^{i w(x)}\left[1+\circ(|\lambda|)^{-\frac{1}{2}}\right]}{i \lambda^{\frac{1}{4}}\left[v_{1}(0) u_{2}(0)-u_{1}(0) v_{2}(0)\right](\lambda-p(x))^{\frac{1}{4}}} \\
=\frac{v_{2}(0) \cdot e^{i w(x)}\left[1+\circ(|\lambda|)^{-\frac{1}{2}}\right]}{i \lambda^{\frac{1}{2}}\left[v_{1}(0) u_{2}(0)-u_{1}(0) v_{2}(0)\right]}\left(1-\frac{p(x)}{\lambda}\right)^{-\frac{1}{4}}
\end{gathered}
$$

Thus from the first result of (3.27) and (6.12), we get

$$
\begin{gathered}
A_{3}=\frac{u_{1}(0) v_{2}(0) \cos w(x)}{i|\lambda|^{\frac{1}{2}}\left[v_{1}(0) u_{2}(0)-u_{1}(0) v_{2}(0)\right]} \int_{x}^{x+\delta} e^{i w(y)} f_{1}(y)\left(1-\frac{P(y)}{\lambda}\right)^{-\frac{1}{4}} d y+ \\
+\mathrm{o}\left\{\frac{e^{|x|}}{|\lambda|} \int_{x}^{x+\delta} e^{-i m(w(y))} f_{1}(y)\left(1-\frac{P(y)}{\lambda}\right)^{-\frac{1}{4}} d y\right\} \\
=\frac{u_{1}(0) v_{2}(0) \cos w(x)}{i|\lambda|^{\frac{1}{2}}\left[v_{1}(0) u_{2}(0)-u_{1}(0) v_{2}(0)\right]} \int_{x}^{x+\delta} e^{i w(y)} f_{1}(y) d y+\mathrm{o}\left\{\frac{e^{|t x|}}{|\lambda|} \int_{x}^{x+\delta} e^{-i m(w(y))} f_{1}(y) d y\right\}+ \\
+\mathrm{o}\left\{\frac{e^{|x|}}{|\lambda|^{\frac{3}{2}}} \int_{x}^{x+\delta} e^{-i m(w(y))} p(y) f_{1}(y) d y\right\}
\end{gathered}
$$

The last two terms of $A_{3}$ are

$$
\mathrm{o}\left\{\frac{1}{|\lambda|} \int_{x}^{x+\delta} f_{1}(y) d y\right\} \text { and } \mathrm{o}\left\{\frac{1}{|\lambda|^{\frac{3}{2}}} \int_{x}^{x+\delta} f(y) p(y) d y\right\} .
$$

The integral of these round the semicircle are o $\left\{\int_{x}^{x+\delta} f_{1}(y) d y\right\}$ and $0\left\{\frac{1}{|\lambda|^{\frac{1}{2}}} \int_{x}^{x+\delta} f(y) p(y) d y\right\}$ respectively. These integrals can be made as small as we please by properly choosing $\delta$ and using (6.1). The first term in $A_{3}$ can be written as

$$
\frac{u_{1}(0) v_{2}(0)\left[e^{i w(x)}+e^{-i w(x)}\right]}{2 i|\lambda|^{\frac{1}{2}}\left[v_{1}(0) u_{2}(0)-u_{1}(0) v_{2}(0)\right]} \int_{x}^{x+\delta} e^{i w(y)} f_{1}(y) d y
$$


Using (3.13), we have from (6.13), the first term of $A_{3}$ is

$$
\frac{u_{1}(0) v_{2}(0)\left[e^{i \mu(x)}+e^{-i \mu(x)}\right]}{2 i \mu\left[v_{1}(0) u_{2}(0)-u_{1}(0) v_{2}(0)\right]} \int_{x}^{x+\delta} e^{i \mu(y)} f_{1}(y) d y
$$

The term involving $e^{i \mu x}$ also gives a zero limit. The other term is the same as in the case of an ordinary Fourier series, and similarly for $A_{2}$. Hence we conclude that in the case of continuous function of bounded variation

Similarly

$$
\lim _{R \rightarrow \infty} \int_{-R+i \varepsilon}^{R+i \varepsilon} A \cdot d \lambda=\frac{\pi i u_{1}(0) v_{2}(0) f_{1}(x)}{v_{1}(0) u_{2}(0)-u_{1}(0) v_{2}(0)}
$$

$$
\begin{aligned}
& \lim _{R \rightarrow \infty} \int_{-R+i \varepsilon}^{R+i \varepsilon} B \cdot d \lambda=-\frac{\pi i v_{1}(0) u_{2}(0) f_{1}(x)}{v_{1}(0) u_{2}(0)-u_{1}(0) v_{2}(0)} \\
& \lim _{R \rightarrow \infty} \int_{-R+i \varepsilon}^{R+i \varepsilon} C \cdot d \lambda=\frac{1}{2} \frac{\pi i v_{2}(0) v_{1}(0) f_{2}(x)}{v_{1}(0) u_{2}(0)-u_{1}(0) v_{2}(0)} \\
& \lim _{R \rightarrow \infty} \int_{-R+i \varepsilon}^{R+i \varepsilon} D \cdot d \lambda=-\frac{1}{2} \frac{\pi i v_{2}(0) v_{1}(0) f_{2}(x)}{v_{1}(0) u_{2}(0)-u_{1}(0) v_{2}(0)} \\
& \lim _{R \rightarrow \infty} \int_{-R+i \varepsilon}^{R+i \varepsilon} E \cdot d \lambda=-\frac{1}{2} \frac{\pi i u_{1}(0) u_{2}(0) f_{2}(x)}{v_{1}(0) u_{2}(0)-u_{1}(0) v_{2}(0)} \\
& \lim _{R \rightarrow \infty} \int_{-R+i \varepsilon}^{R+i \varepsilon} F \cdot d \lambda=\frac{1}{2} \frac{\pi i u_{1}(0) u_{2}(0) f_{2}(x)}{v_{1}(0) u_{2}(0)-u_{1}(0) v_{2}(0)}
\end{aligned}
$$

Thus we have

$$
f_{1}(x)=-\frac{1}{\pi i} \lim _{R \rightarrow \infty} \int_{-R+i \varepsilon}^{R+i \varepsilon} \varphi_{1}(x, \lambda) d \lambda
$$

Similarly

$$
f_{2}(x)=-\frac{1}{\pi i} \lim _{R \rightarrow \infty} \int_{-R+i \varepsilon}^{R+i \varepsilon} \varphi_{2}(x, \lambda) d \lambda
$$

The above results are true, uniformly for $0<\varepsilon \leq 1$.

\section{References}

[1]. Bhagat, B.'Eigen function expansions associated with a pair of second order differential equations.'Proc. National Inst. Sciences of India. Vol. 35,A, No. 1 (1969)

[2]. Bhagat, B.'Some problems on a pair of singular second order differential equations' Ibid. 35A (1969), $232-244$

[3]. Bhagat, B.'A spectral theorem for a pair of second order singular differential equations' Quart. J. Math. Oxford 21, (1970), 487-495.

[4]. Conte, S. D.andSangren, W.C.'On asymptotic solution for a pair of singular first order equations' Proc. Amer. Math. Soc. 4, (1953) 696-702

[5]. Pandey, Y.P.'Some problems on eigenfunction expansion associated with a pair of second order differential equations.' Thesis, Bhag. Univ. Bihar

[6]. Pandey, Y. P.and Kumar, A. 'Spectral theorem and eigenfunction expansion associated with a pair of second order differential equations.' Thesis, T. M. Bhag. Univ. , Bihar

[7]. Rose, B. W.andSangren, W. C.'Expansions associated with a pair of a singular first order differential equations' J. Math. Physics 4 (1963) 999-1008.

[8]. Titchmarsh, E.C.'Eigenfunction expansions associated with second order differential equation' Part I, Oxford 1962 